\title{
Serum zonulin levels in type 2 diabetes patients with diabetic kidney disease
}

\author{
Fevziye Burcu Sirin ${ }^{\circledR 1}$, Hakan Korkmaz ${ }^{\circledR 2}$, Ismet Eroglu ${ }^{\circledR 3}$, Barıs Afsar ${ }^{\circledR 4}$, Duygu Kumbul Doguc ${ }^{(101}$ \\ ${ }^{1}$ Department of Medical Biochemistry, Faculty of Medicine, Süleyman Demirel University, Isparta, Turkey \\ ${ }^{2}$ Division of Endocrinology and Metabolism, Department of Internal Medicine, Faculty of Medicine, Süleyman Demirel University, \\ Isparta, Turkey \\ ${ }^{3}$ Department of Internal Medicine, Isparta State Hospital, Isparta, Turkey \\ ${ }^{4}$ Division of Nephrology, Department of Internal Medicine, Faculty of Medicine, Süleyman Demirel University, Isparta, Turkey
}

\begin{abstract}
Introduction: Recent data have shown that diabetic kidney disease (DKD) is associated with abnormal gut microbiota composition. Zonulin is a physiological tight junction modulator and an intestinal permeability marker. In this study we aimed to investigate serum levels of zonulin and interleukin 6 (IL-6) in patients with type 2 diabetes mellitus (T2DM) and different levels of albuminuria.

Material and methods: Ninety patients with T2DM and 30 healthy controls (HC) aged between 18 and 65 years were enrolled in the study. T2DM patients were divided into three groups as patients with normoalbuminuria $(\mathrm{n}=30)$, microalbuminuria $(\mathrm{n}=30)$, and macroalbuminuria $(\mathrm{n}=30)$. Serum zonulin and IL-6 levels were measured by ELISA method.

Results: There was no significant difference between groups in terms of age, gender, serum ALT, LDL-C, HDL-C, and zonulin levels $(p>0.05)$. Significant differences between groups were present for the duration of diabetes $(p<0.001)$, body mass index $(p<0.001)$, fasting blood glucose $(p<0.001)$, creatinine $(p<0.001)$, uric acid $(p=0.037)$, triglyceride $(p=0.003)$, total cholesterol $(p<0.001)$, glycated haemoglobin ( $p<0.001)$, and IL-6 ( $<<0.001)$ levels. IL-6 levels were significantly increased in the microalbuminuria and macroalbuminuria groups compared to the HC group, but no significant difference was determined between the HC and normoalbuminuria group. In patients with diabetic kidney disease, a significant positive correlation was found between zonulin with IL- 6 and proteinuria (rho $=0.296$, $\mathrm{p}=0.008 ;$ rho $=0.190, \mathrm{p}<0.047$, respectively). The serum IL-6 level was positively correlated with microalbuminuria and proteinuria (rho $=0.451, \mathrm{p}<0.001 ;$ rho $=0.425, \mathrm{p}<0.001$, respectively).

Conclusions: We suggest that the serum zonulin level is not a promising biomarker to assess the severity of DKD in patients with longstanding T2DM. (Endokrynol Pol 2021; 72 (5): 545-549)
\end{abstract}

Key words: type 2 diabetes mellitus; diabetic kidney disease; intestinal permeability; zonulin; inflammation

\section{Introduction}

Diabetic kidney disease (DKD), one of the major microvascular complications of diabetes mellitus (DM), is the leading cause of chronic kidney disease (CKD) and end-stage renal disease. DKD develops in approximately one-third of all DM patients [1]. The pathogenesis of DKD is a multifactorial and complex process. Hyperglycaemia, hypertension, hyperlipidaemia, obesity, ethnicity, and genetic predisposition are responsible for DKD development and progression [2]. In addition, inflammation together with oxidative stress and fibrosis are also key factors in the progression of DKD [3]. Recently, intestinal microbiota was blamed for chronic inflammation in DM and chronic kidney patients [4-5].

CKD patients may have altered intestinal microbiota composition (dysbiotic gut) due to the slowed intestinal transit time, decreased digestive capacity, and secretion of ammonia and urea into the gut [4]. Recent evidence suggests that this dysbiotic gut causes production of major uremic toxins such as indoxyl sulphate, p-cresyl sulphate, and trimethylamine-N-oxide, which might be linked to the progression of CKD [2]. On the other hand, gut microbiota alteration was shown to affect the development of proteinuria, hypertension, inflammation, and diabetes mellitus. In this respect, it is thought that there is a vicious cycle between CKD formation and gut microbiota [6].

Zonulin is a $47 \mathrm{kDa}$ protein, which is encoded by the haptoglobin gene and regulates paracellular transport by modulation of enterocyte tight junctions. Upregulation of zonulin by triggering factors (gluten, increased fatty acid, etc.) leads to disassembly of tight junctions, uncontrolled antigen trafficking from the intestinal lu- 
men to submucosa, and consequently altered intestinal permeability, which gives rise to chronic inflammatory diseases [7-8]. Zonulin expression is suggested to be controlled by a proinflammatory cytokine interleukin 6 (IL-6) via STAT3 activation and miR-18a induction [9]. In recent years various studies have shown that circulatory zonulin levels are elevated in several metabolic and inflammatory diseases such as insulin resistance, obesity, metabolic syndrome, T1DM, and T2DM [10-13].

In this study we aimed to evaluate serum levels of zonulin as an intestinal permeability marker and IL-6 as an inflammation marker in relatively long-standing T2DM patients with and without DKD, to investigate correlations with metabolic and biochemical parameters.

\section{Material and methods}

This cross-sectional study was approved by the Ethics Committee of the Faculty of Medicine, Suleyman Demirel University, Isparta, Turkey (16.04.2019-137) and conducted in accordance with the Helsinki Declaration. All participants gave verbal and written informed consent prior to participation in the study.

\section{Patients and control subjects}

A total of 120 participants (78 female, 42 male) between the ages of 18 and 65 years were recruited in this study from the Endocrine and Metabolism Outpatient Clinic of Suleyman Demirel University Research and Practice Hospital. A healthy control group of 30 subjects without T2DM $(\mathrm{HC} ; \mathrm{n}=30)$ and 90 patients with T2DM were included in the study. Type 2 diabetic patients were grouped according to urinary albumin as normoalbuminuria ( $<30 \mathrm{mg} / 24 \mathrm{hour}$; $\mathrm{n}=30$ ), microalbuminuria ( $30-300 \mathrm{mg} / 24$ hour; $\mathrm{n}=30$ ), and macroalbuminuria groups $(>300 \mathrm{mg} / 24$ hour; $\mathrm{n}=30)$. Exclusion criteria included the presence of T1DM, pregnancy, acute or chronic infectious disease, rheumatological disease, hepatic disease, gastrointestinal disease, cancer, and regular usage of anti-inflammatory drugs. The HC group was randomly selected from people without any systemic disease or drug use. At the time of recruitment, the body weight and height of all participants were measured, and body mass index (BMI) was calculated as weight/height ${ }^{2}$ and expressed in kilograms per square metre $\left(\mathrm{kg} / \mathrm{m}^{2}\right)$. Systolic/diastolic blood pressure measurements were also performed. Blood pressure measurements were performed according to European Society of Cardiology (ESC)-European Society of Hypertension (ESH) guidelines on hypertension [14]. Age, sex, time since diagnosis of $\mathrm{DM}$ (duration of DM), and existence of micro- and macrovascular complications of T2DM were also recorded.

\section{Sample collection and biochemical measurements}

All biochemical analyses were performed at the Medical Biochemistry Laboratory of Suleyman Demirel University Research and Practice Hospital. Venous blood samples were collected after a minimum of eight hours fasting for glycated haemoglobin $\left(\mathrm{HbA}_{1 \mathrm{c}}\right)$, routine chemistry analysis, and zonulin and IL- 6 measurements. Also, 24-hour urine samples were collected by participants for urinary protein, microalbumin, and creatinine measurement. HbA1c was determined by high-performance liquid chromatography method (Bio-Rad Co, CA, USA) from blood collection tubes containing EDTA. Serum specimens were separated after centrifuge of clot activator blood collection tubes at $3000 \mathrm{rpm}$ for 10 minutes. On the same day of the blood collection, serum fasting blood glucose, alanine aminotransaminase (ALT), creatinine, uric acid, triglyceride (TG), high-density lipoprotein cholesterol (HDL-C), total cholesterol (TC), and 24-hour urine protein and albumin were measured on a Beck- man Coulter AU 5800 chemistry analyser (Beckman Coulter, Brea, USA). The Friedewald formula: $L D L-C=T C-(H D L-C+(T G / 5))$, was used for low-density lipoprotein cholesterol (LDL-C) calculation, and the glomerular filtration rate (GFR) was estimated with the Chronic Kidney Disease Epidemiology Collaboration (CKD-EPI) equation [15]. Serum samples were portioned into Eppendorf tubes and frozen at $-80^{\circ}$ until analysis of serum zonulin and IL- 6 levels. Commercial human enzyme-linked immunosorbent assay (ELISA) kits were used for the determination of serum zonulin and IL-6 levels (E-EL-H5560, E-EL-H0102; Elabscience, Wuhan, China). Detection limits of the ELISA tests were $0.47 \mathrm{ng} / \mathrm{mL}$ and $4.69 \mathrm{pg} / \mathrm{mL}$, respectively.

\section{Statistical analyses}

All statistical analyses were performed using SPSS version 22 (SPSS Inc., Chicago, IL, USA). The $\chi^{2}$ test was used to compare categorical data. The continuous variables were investigated using the ShapiroWilk normality test. Parameters with normal distribution were compared with one-way analysis of variance (ANOVA) followed by Tukey HSD post-hoc test, and parameters with non-normal distribution were compared using the Kruskal-Wallis test followed by Mann-Whitney U test. Correlations between parameters were evaluated with Spearman's correlation analysis. A p value of less than 0.05 was considered to show a statistically significant result.

\section{Results}

A total of 90 T2DM patients (30 patients with normoalbuminuria, 30 patients with microalbuminuria, 30 patients with macroalbuminuria) and $30 \mathrm{HC}$ were included in this study. Demographic, clinical, and laboratory parameters and statistical results for comparisons of the groups are shown in Table 1. There was no significant difference between groups for age, gender, serum ALT, LDL-C, HDL-C, and zonulin levels ( $p>0.05$ ). Significant differences between groups were present for duration of diabetes ( $p<0.001)$, BMI $(p<0.001)$, fasting blood glucose $(p<0.001)$, creatinine $(p<0.001)$, uric acid $(p=0.037)$, TG $(p=0.003)$, TC $(p<0.001), H_{b A}(p<0.001)$, and IL-6 $(p<0.001)$ levels. IL-6 levels were significantly increased in both micro and macro albuminuria groups of DKD patients compared to the HC group, but no significant difference was determined between $\mathrm{HC}$ and normoalbuminuria group (Tab. 1, Fig. 1 and 2). In patients with DKD, a significant positive correlation was found between zonulin with IL-6 and proteinuria (rho $=0.296, \mathrm{p}=0.008$; rho $=0.190, p=0.047$, respectively). Serum IL-6 level was positively correlated with albuminuria and proteinuria (rho $=0.451, \mathrm{p}<0.001 ;$ rho $=0.425, \mathrm{p}<0.001$ ).

There was no significant difference between the use of antidiabetic (except insulin) and antihyperlipidemic medications among diabetic patients. Regarding medications, most of the patients $(74 \%)$ were taking metformin, and approximately half of the patients (46\%) were on insulin therapy. Other medications and the percentages of patients using them were as follows; glucagon-like peptide (GLP) analogues or dipeptidyl peptidase 4 (DPP4) inhibitors $(45 \%)$, sulphonylurea $(26 \%)$, sodium-glucose co-transporter-2 (SGLT2) inhibitor $(4.4 \%)$, glitazone $(4.4 \%)$, and antihyperlipidemic (36\%) agents. 
Table 1. Demographic, clinical, and biochemical characteristics of the groups $(n=120)$

\begin{tabular}{|c|c|c|c|c|c|}
\hline & Healthy control & $\begin{array}{c}\text { Normo } \\
\text { albuminuria }\end{array}$ & $\begin{array}{c}\text { Micro } \\
\text { albuminuria }\end{array}$ & $\begin{array}{c}\text { Macro } \\
\text { albuminuria }\end{array}$ & p value \\
\hline Gender [male/female] & $20 / 10$ & $18 / 12$ & $20 / 10$ & $20 / 10$ & 0.932 \\
\hline Age [years] & $56.0 \pm 5.9$ & $53.5 \pm 8.1$ & $54.8 \pm 6,6$ & $55.5 \pm 8.2$ & 0.564 \\
\hline Duration [years] & 0 & $8.4 \pm 6.6^{\mathrm{a}}$ & $7.7 \pm 6.2^{\mathrm{a}}$ & $15.3 \pm 8.5^{\mathrm{a}-\mathrm{c}}$ & $<0.001$ \\
\hline BMI $\left[\mathrm{kg} / \mathrm{m}^{2}\right]$ & $28.1 \pm 3.6$ & $30.4 \pm 4.6$ & $33.1 \pm 5.6^{a}$ & $33.3 \pm 7.2^{\mathrm{a}}$ & $<0.001$ \\
\hline FBG [mg/dL] & $93.5(11)$ & $156(80.5)^{\mathrm{a}}$ & $157(93.5)^{\mathrm{a}}$ & $199.5(149.5)^{a, b}$ & $<0.001$ \\
\hline $\mathrm{HbA}_{1 \mathrm{c}}(\%)$ & $5.5(0.5)$ & $7.6(2.4)^{\mathrm{a}}$ & $8.2(3.9)^{\mathrm{a}}$ & $9.6(4.4)^{\mathrm{a}}$ & $<0.001$ \\
\hline ALT [mg/dL] & $18.5(9.5)$ & $19.5(14.5)$ & $21.0(12.0)$ & $19.0(8.5)$ & 0.167 \\
\hline $\mathrm{TG}[\mathrm{mg} / \mathrm{dL}]$ & $109.5(83)$ & $138.0(108.8)$ & $141.5(65.0)$ & $181.0(267.3)^{a-c}$ & 0.003 \\
\hline $\mathrm{TC}[\mathrm{mg} / \mathrm{dL}]$ & $209.5(59.8)$ & $212.5(45.0)$ & $195.5(44.0)$ & $245.2(105.5)^{a-c}$ & $<0.001$ \\
\hline LDL-C [mg/dL] & $134.0 \pm 29.4$ & $124.6 \pm 24.5$ & $116.2 \pm 30$ & $131.6 \pm 51.3$ & 0.206 \\
\hline HDL-C [mg/dL] & $48.5(16.0)$ & $48.0(18.5)$ & $44.0(11.0)$ & $45.5(20.0)$ & 0.060 \\
\hline Uric acid [mg/dL] & $4.9(2.1)$ & $5.0(1.3)$ & $5.2(2.4)$ & $6.5(1.9)^{a-c}$ & 0.037 \\
\hline $\mathrm{Cr}[\mathrm{mg} / \mathrm{dL}]$ & $0.85(0.25)$ & $0.88(0.17)$ & $0.90(0.15)$ & $1.15(0.53)^{a-c}$ & $<0.001$ \\
\hline GFR [ml/min] & $92.0(10.8)$ & $85.5(28.5)$ & $86.0(32.8)$ & $74.0(40.8)^{\mathrm{a}-\mathrm{c}}$ & $<0.001$ \\
\hline Protein [g/24 h] & $0.08(0.04)$ & $0.10(0.05)$ & $0.19(0.16)^{a, b}$ & $1.30(2.3)^{\mathrm{a}-\mathrm{c}}$ & $<0.001$ \\
\hline Alb [mg/24 h] & $2.35(5.85)$ & $9.8(10.2)^{\mathrm{a}}$ & $64.5(80.8)^{\mathrm{a}, \mathrm{b}}$ & $678.5(685.8)^{a-c}$ & $<0.001$ \\
\hline Zonulin [ng/mL] & $101.8(115.5)$ & $107.1(101.9)$ & $115.4(75.0)$ & $116.5(156.8)$ & 0.580 \\
\hline IL-6 [pg/mL] & $5.02(11.0)$ & $5.03(10.3)$ & $14.08(12.6)^{\mathrm{a}, \mathrm{b}}$ & $16.8(13.7)^{\mathrm{a}, \mathrm{b}}$ & $<0.001$ \\
\hline
\end{tabular}

Normal distributed data are given as mean $\pm \mathrm{SD}$, non-normal distributed data are given as median (IQR). Bold values are statistically significant ( $p<0.05)$. ${ }^{a}$ significant

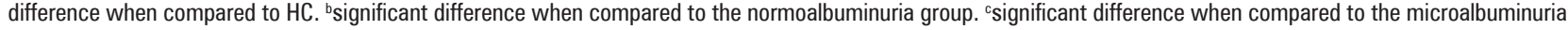
group. BMI — body mass index; FBG — fasting blood glucose; TG — triglyceride; TC — total cholesterol; LDL-C — low-density lipoprotein; HDL-C — high-density lipoprotein; $\mathrm{Cr}$ — creatinine; GFR — glomerular filtration rate; Alb — albumin; IL-6 — interleukin 6

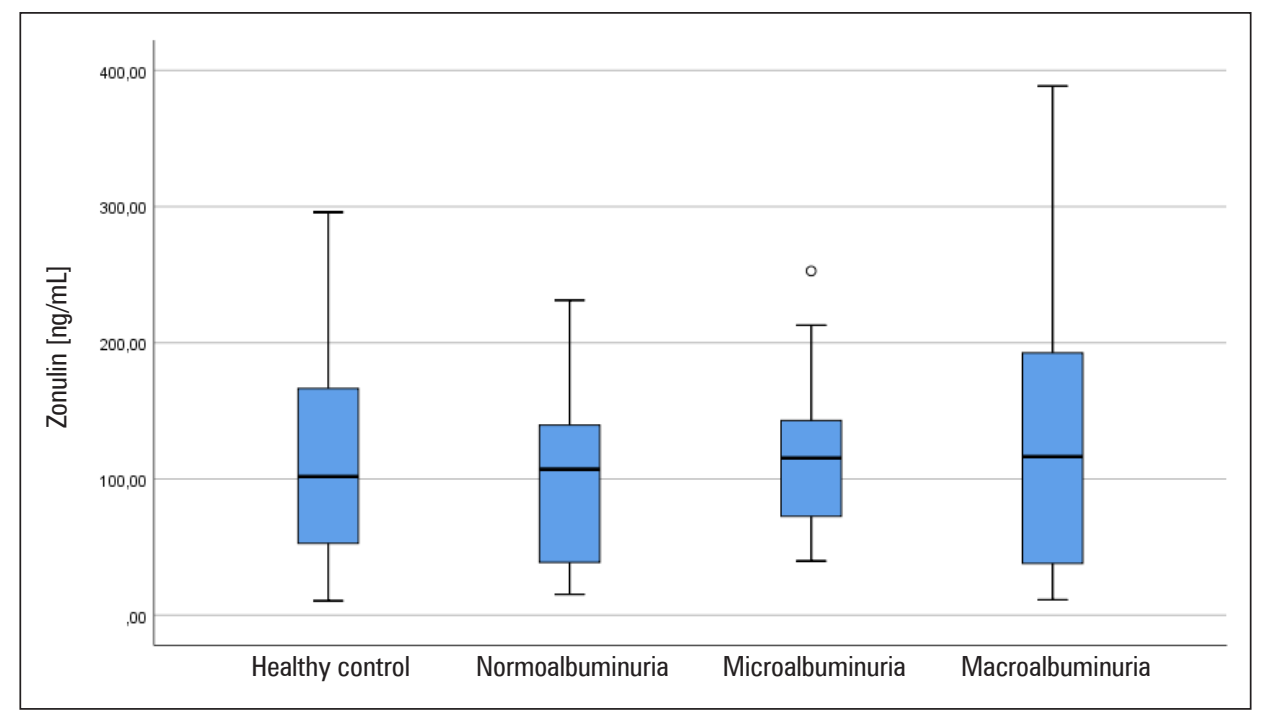

Figure 1. Serum zonulin levels in study groups

\section{Discussion}

The present study assessed the serum zonulin and IL-6 levels in cases with long history of T2DM and their possible association with the presence and severity of albuminuria. Although serum IL-6 levels were significantly increased in DKD patients when compared to both $\mathrm{HC}$ and normoalbuminuria groups, the zonulin levels were not significantly different between the groups. Interleukin 6 levels were simultaneously increased with the progression of DKD, which may represent the role of chronic inflammation in the pathogenesis of DKD. 


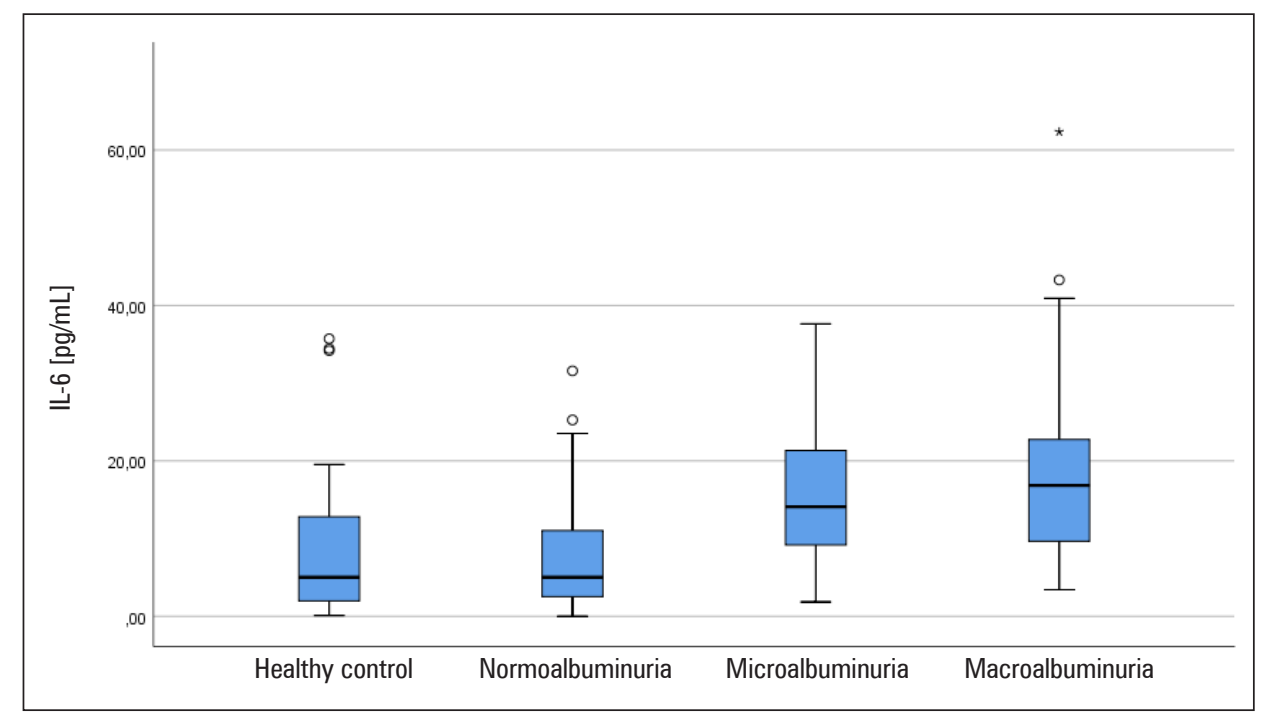

Figure 2. Serum interleukin 6 (IL-6) levels in study groups

In the literature, two studies that evaluated newly diagnosed T2DM patients showed increased serum zonulin levels $[12,13]$. Zhang et al. showed a progressive increase in serum zonulin level with the impairment of glucose tolerance and significantly high levels in DM patients [12]. Jayashree et al. found that serum levels of lipopolysaccharide, IL-6, tumour necrosis factor-alpha, and zonulin were significantly increased in the newly diagnosed T2DM group compared to the healthy group [13]. In our study significant increases in IL-6 levels were observed in the micro- and macroalbuminuria (DKD positive) groups. On the other hand, although the zonulin levels tended to increase with the progression of DKD, the difference was not significant between the groups. Our results are partly consistent with the literature because the IL-6 levels simultaneously increased with the progression of DM. Our findings regarding zonulin contradict the studies of Zhang et al. and Jayashree et al., and this contradiction may be due initially to the duration of diabetes as our diabetic patients were diagnosed for a relatively long period and prescribed antidiabetic drugs for that period, but the diabetic groups in the mentioned studies were newly diagnosed. Secondly, human gut microbiota composition is extremely variable between individuals, and therapies such as metformin usage and different eating habits were shown to restructure gut microbiota [16, 17].

There are several studies that reported abnormal gut microbiota in uncomplicated and complicated T1DM patients with advanced stages of DKD. Winther et al. reported significant differences in both gut microbiota and plasma metabolome according to the severity of DKD [18]. In an experimental study, it was shown that phenyl sulphate, originating from the gut microbiota of rats, causes diabetic kidney disease in rats. When tyrosine phenol-lyase enzyme, which ensures the formation of phenyl sulphate, is inhibited albuminuria was shown to decrease [19].

In another study, performed by Al-Obaide et al., serum trimethylamine-N-oxide, lipopolysaccharide, zonulin, and IL-6 levels were shown to be increased in T2DM patients with advanced kidney disease [glomerular filtration rate (GFR) $<30 \mathrm{~mL} / \mathrm{min}$ ] compared to healthy controls [20]. In our study the T2DM patients were classified by their albuminuria levels, but the GFR levels in our groups were mildly impaired because macroalbuminuria group had the lowest median GFR value of $74 \mathrm{~mL} / \mathrm{min}$ and the highest serum creatinine levels with a median value of $1.15 \mathrm{mg} / \mathrm{dL}$ (Tab. 1).

In a study by Hasslatcher et al. 63 diabetic patients were studied with a mean of 13 years duration. Zonulin levels of diabetic patients were higher than healthy controls, and zonulin concentrations were correlated with $\mathrm{BMI}$ and $\mathrm{HbA}_{1 \mathrm{c}}$ [21]. Contrary to this study, we found no significant difference between the zonulin levels of healthy control and 90 T2DM patients with a mean of 10 years duration. In our study zonulin levels correlated only with IL-6 and urinary protein excretion, but there was no correlation between the other factors (GFR, creatinine, BMI). Another study by Dschietzig et al. speculated that the plasma zonulin level can decrease with urine excretion and observed inverse associations of zonulin with creatinine in T2DM. They claimed that urinary zonulin excretion may increase in parallel with the severity of DKD because zonulin is a smaller protein than albumin [22]. In our study, we did not measure zonulin excretion in urine, but there was a positive relationship between the intensity of protein excretion and serum zonulin levels, although it did not reach statistical significance between the groups. 
In our study, when diabetics with mainly albuminuria (micro- and macroalbuminuria) $(\mathrm{n}=60)$ and the healthy group were evaluated in terms of zonulin and IL-6 levels, there was no difference in zonulin, but IL-6 levels increased significantly. The IL-6 levels were positively correlated with blood glucose, $\mathrm{HbA}_{1 c^{\prime}} \mathrm{BMI}$, TG, creatinine levels, urinary albumin, and protein excretion and negatively correlated with GFR. As anticipated, during DKD progression the level of fasting glucose, $\mathrm{HbA}_{1 c^{\prime}}$ and creatinine levels steadily increased and GFR decreased over the years due to the long-term exposure to high glucose levels increasing the risk of vascular damage to kidney cells. Significantly higher levels of IL-6 were found in the macroalbuminuria group compared to the $\mathrm{HC}$ and normoalbuminuria groups; however, inflammation and diabetic kidney disease progression was not related with serum zonulin concentration.

Our study has certain limitations. First, we did not assess the microbiota composition. Second, uremic toxin analysis was not performed, and third, the absence of dietary information of patients is an additional limitation.

\section{Conclusions}

We suggest that the serum zonulin level is not a promising biomarker to assess the severity of DKD in patients with long-standing T2DM.

\section{References}

1. Rabkin R. Diabetic nephropathy. Clinical Cornerstone. 2003; 5(2): 1-11, doi: 10.1016/s1098-3597(03)90014-7, indexed in Pubmed: 12800476.

2. Fernandes R, Viana SD, Nunes S, et al. Diabetic gut microbiota dysbiosis as an inflammaging and immunosenescence condition that fosters progression of retinopathy and nephropathy. Biochim Biophys Acta Mol Basis Dis. 2019; 1865(7): 1876-1897, doi: 10.1016/j.bbadis.2018.09.032, indexed in Pubmed: 30287404

3. Wada J, Makino H. Inflammation and the pathogenesis of diabetic nephropathy. Clin Sci(Lond). 2013; 124(3): 139-152, doi: 10.1042/CS20120198, indexed in Pubmed: 23075333.

4. Wing MR, Patel SS, Ramezani A, et al. Gut microbiome in chronic kidney disease. Exp Physiol. 2016; 101(4): 471-477, doi: 10.1113/EP085283, indexed in Pubmed: 26337794.

5. Khoury T, Tzukert K, Abel R, et al. The gut-kidney axis in chronic renal failure: A new potential target for therapy. Hemodial Int. 2017; 21(3): 323-334, doi: 10.1111/hdi.12486, indexed in Pubmed: 27634464.

6. Kanbay M, Onal EM, Afsar B, et al. The crosstalk of gut microbiota and chronic kidney disease: role of inflammation, proteinuria, hyperten- sion, and diabetes mellitus. Int Urol Nephrol. 2018; 50(8): 1453-1466, doi: 10.1007/s11255-018-1873-2, indexed in Pubmed: 29728993

7. Fasano A, Not T, Wang W, et al. Zonulin, a newly discovered modulator of intestinal permeability, and its expression in coeliac disease. Lancet. 2000; 355(9214): 1518-1519, doi: 10.1016/S0140-6736(00)02169-3, indexed in Pubmed: 10801176.

8. Fasano A. Zonulin and its regulation of intestinal barrier function: the biological door to inflammation, autoimmunity, and cancer. Physio Rev. 2011; 91(1): 151-175, doi: 10.1152/physrev.00003.2008, indexed in Pubmed: 21248165.

9. Brock M, Trenkmann M, Gay RE, et al. MicroRNA-18a enhances the interleukin-6-mediated production of the acute-phase proteins fibrinogen and haptoglobin in human hepatocytes. J Biol Chem. 2011; 286(46): 40142-40150, doi: 10.1074/jbc.M111.251793, indexed in Pubmed: 21953462

10. Moreno-Navarrete JM, Sabater M, Ortega F, et al. Circulating zonulin a marker of intestinal permeability, is increased in association with obesity-associated insulin resistance. PLoS One. 2012; 7(5): e37160, doi: 10.1371/journal.pone.0037160, indexed in Pubmed: 22629362.

11. Sapone A, de Magistris L, Pietzak M, et al. Zonulin upregulation is associated with increased gut permeability in subjects with type 1 diabetes and their relatives. Diabetes. 2006; 55(5): 1443-1449, doi: 10.2337/db05-1593, indexed in Pubmed: 16644703.

12. Zhang D, Zhang L, Zheng $\mathrm{Y}$, et al. Circulating zonulin levels in newly diagnosed Chinese type 2 diabetes patients. Diabetes Res Clin Pract. 2014; 106(2): 312-318, doi: 10.1016/j.diabres.2014.08.017, indexed in Pubmed: 25238913

13. Jayashree B, Bibin YS, Prabhu D, et al. Increased circulatory levels of lipopolysaccharide (LPS) and zonulin signify novel biomarkers of proinflammation in patients with type 2 diabetes. Mol Cell Biochem. 2014; 388(1-2): 203-210, doi: 10.1007/s11010-013-1911-4 indexed in Pubmed: 24347174.

14. Williams B, Mancia G, Spiering W, et al. ESC Scientific Document Group . 2018 ESC/ESH Guidelines for the management of arterial hypertension. Eur Heart J. 2018; 39(33): 3021-3104, doi: 10.1093/eurheartj/ehy339, indexed in Pubmed: 30165516.

15. Levey AS, Stevens LA, Schmid CH, et al. CKD-EPI (Chronic Kidney Disease Epidemiology Collaboration). A new equation to estimate glomerular filtration rate. Ann Intern Med. 2009; 150(9): 604-612, doi: 1 0.7326/0003-4819-150-9-200905050-00006, indexed in Pubmed: 19414839.

16. Eckburg PB, Bik EM, Bernstein CN, et al. Diversity of the human intestinal microbial flora. Science. 2005; 308(5728): 1635-1638, doi: 10.1126/science.1110591, indexed in Pubmed: 15831718.

17. Ahmadi S, Razazan A, Nagpal R, et al. Metformin Reduces Aging-Related Leaky Gut and Improves Cognitive Function by Beneficially Modulating Gut Microbiome/Goblet Cell/Mucin Axis. J Gerontol A Biol Sci Med Sci. 2020; 75(7): e9-ee21, doi: 10.1093/gerona/glaa056, indexed in Pubmed: 32129462.

18. Winther SA, Henriksen P, Vogt JK, et al. Gut microbiota profile and selected plasma metabolites in type 1 diabetes without and with stratification by albuminuria. Diabetologia. 2020; 63(12): 2713-2724, doi: 10.1007/s00125-020-05260-y, indexed in Pubmed: 32886190.

19. Kikuchi K, Saigusa D, Kanemitsu Y, et al. Gut microbiome-derived phenyl sulfate contributes to albuminuria in diabetic kidney disease. $\mathrm{Na}$ Commun. 2019; 10(1): 1835, doi: 10.1038/s41467-019-09735-4, indexed in Pubmed: 31015435.

20. Al-Obaide MAI, Singh R, Datta P, et al. Gut Microbiota-Dependent Trimethylamine-N-oxide and Serum Biomarkers in Patients with T2DM and Advanced CKD. J Clin Med. 2017; 6(9): 86, doi: 10.3390/jcm6090086, indexed in Pubmed: 28925931.

21. Hasslacher C, Kulozik F, Platten I. Serum zonulin as a parameter of intestinal permeability in longstanding type 2 diabetes: correlation with metabolism parameter and renal function. J Diabetes Metab Disord Control. 2018; 5(2): 58-62, doi: 10.15406/jdmdc.2018.05.00138

22. Dschietzig TB, Boschann F, Ruppert J, et al. Plasma Zonulin and its Association with Kidney Function, Severity of Heart Failure, and Metabolic Inflammation. Clin Lab. 2016; 62(12): 2443-2447, doi: 10.7754/Clin. Lab.2016.160512, indexed in Pubmed: 28164545 